\title{
SURGICAL TREATMENT FOR EYELID DEFORMITY IN CROUZON SYNDROME ASSOCIATED WITH ACANTHOSIS NIGRICANS: CASE REPORT
}

Henri Friedhofer, Alan M. W. Ocharan, Gustavo P. Sturtz, Alexandre S. F. Fonseca, Pedro S. Coltro, and Marcus C. Ferreira

The combination of Crouzon syndrome with acanthosis nigricans is unusual. The incidence of this combination is still uncertain, and there are only 30 cases reported in the medical literature. ${ }^{1-3}$ Crouzon syndrome is caused by mutation of the gene FGFR3 (fibroblast growth factor receptor 3). In this gene, other mutations can cause a kind of nanism that is lethal during the first weeks of life. Recent biomolecular studies have indicated that the association of Crouzon syndrome with acanthosis nigricans should be considered as a different pathology, as opposed to Crouzon syndrome in isolation, because acanthosis nigricans would originate from a different gene and from a diverse chromosome mutation. ${ }^{4}$ Crouzon syndrome is an autosomal dominant disease that involves craniosynostosis, exophthalmia, hypoplasia of the middle third of the face, and irregularities of dental occlusion. Its incidence is about 1 in 300,000 live births.

Acanthosis nigricans is a rare illness related to the skin. It is characterized by hyperplasia and hypertrophy, with hyperkeratosis and slight hyperpigmentation on the inner skin layer, without melanocytic hyperplasia. The epidermis presents as a wavy surface (Figure 2). It can affect the neck, armpits, normally folding areas, and the orbital region. The etiology of acanthosis nigricans is variable and can be one of the following:

- Hereditary with dominant autosomal transmission.

- Associated with genetic abnormalities or endocrine diseases.

- Pseudo-acanthosis nigricans resulting from complications of obesity.

- Induced by medications.

- Combined with cancer, usually gastrointestinal adenocarcinoma.

The association of acanthosis nigricans with Crouzon syndrome creates a phenotypic pattern. All patients that

Division of Plastic Surgery, São Paulo University Medical School - São Paulo/ SP.

Email: alexandrefonseca@terra.com.br have been described in medical literature have had an excess of skin and hyperpigmented lesions in the orbital area, predominantly on the lower eyelid, and on the peroral area, neck, and armpits

\section{CASE REPORT}

The patient, a 25-year-old woman, presented with a skin excess and hyperpigmentation on the lower eyelid. These alterations had started during her teenage years. Crouzon syndrome was diagnosed after her birth, and when she was 3 years old, she underwent craniosynostosis correction. By the time of the physical exam, the patient presented exophthalmia, hypertelorism, craniofacial deformities, hypoplasia of the middle third of the face, intense hyperpigmentation of the armpits, cervical region, oral, nasolabial, and upper and lower eyelid creases. The skin was irregular and thick. (Figure 1). The lower eyelid fissures were oblique, and there was skin excess on the lower and upper eyelid on both sides, primarily on the lower eyelids. The lateral retinaculum was medially displaced, and the upper eyelids were $4 \mathrm{~mm}$ below the scleral edge. These are characteristics of a slight eyelid ptosis associated with telecanthus. The adipose bags were more evident on the lower eyelids (Figure 1).

A cutaneous blepharoplasty was performed through resection of skin and preaponeurotic fat (resulting in additional soft-tissue reduction and volume depletion), and bilateral canthal suspension was performed (Figure 3). The lateral retinaculum was sutured with Ethibond ${ }^{\mathrm{TM}} 4-0$ to the reversed border of the periosteum. The continuous Mononylon ${ }^{\mathrm{TM}}$ 6-0 cutaneous suture was removed 5 days later. The scars healed in a widened and depressed pattern on the upper eyelid and on the lateral lower portion.

Microscopic histological examination revealed hyperkeratosis of the epidermis, acanthosis with widening of the epithelial cones, and papillomatosis, which can be described as acanthosis nigricans (Figure 2). The orbicular 
muscular tissue and the periorbital fat did not exhibit histological alterations.

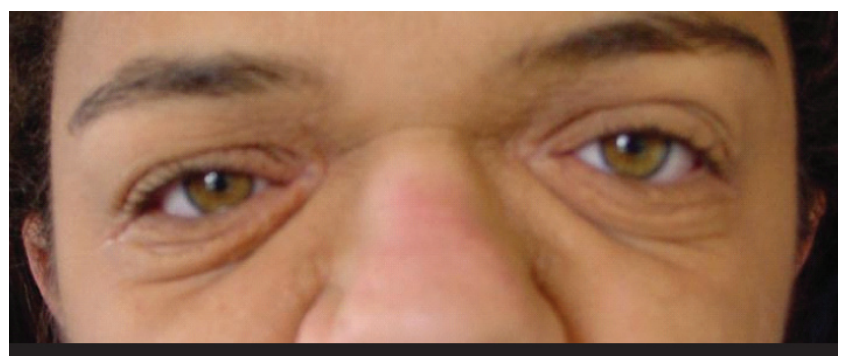

Figure 1 - Preoperative view showing a cutaneous excess on the lower eyelid before resection of the upper eyelid skin and lateral canthal suspension

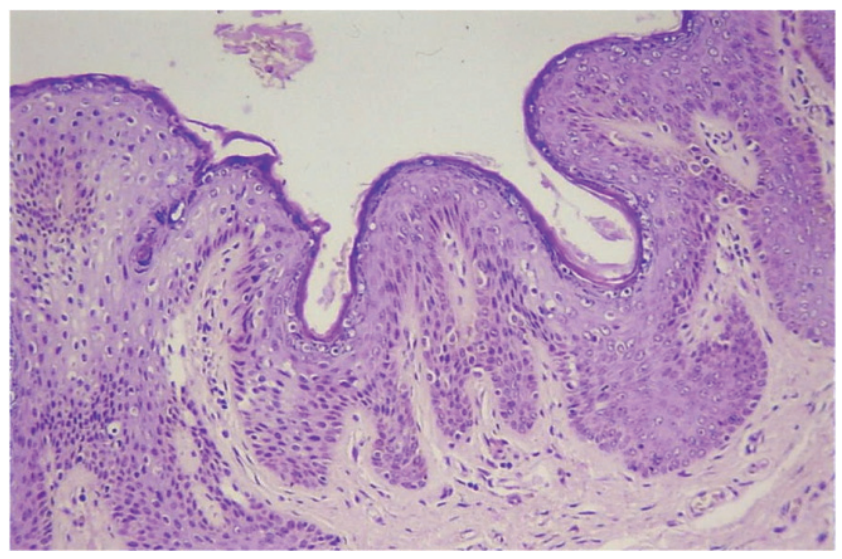

Figure 2 - The hyperplasia of the corneal extract can be seen in greater detail along with an increased number of keratinocytes, an increase of the papillary dermis, and an extension of the dermal papillae. These alterations are characteristics of acanthosis nigricans (hemotoxylin and eosin 50x)

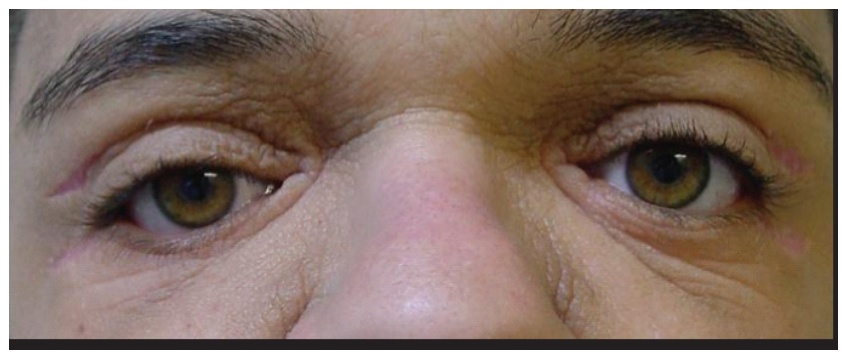

Figure 3 - Final results after 1 year. Lateral canthal suspension provided a better lower eyelid position because it pulled and suspended its components, and it especially stabilized the lower eyelid with the orbit periosteum

\section{DISCUSSION}

Some characteristics of the association of Crouzon syn- drome and acanthosis nigricans differ from cases of isolated Crouzon syndrome. There is a greater frequency of isolated Crouzon syndrome occurring in men, while the association of Crouzon syndrome with acanthosis nigricans occurs more frequently in women, at a 9:2 ratio. The phenotype of these patients is very similar, with skin wrinkles and hyperpigmentation of the orbital region. When these characteristics occur in combination with Crouzon syndrome, the patients look much more alike one another. In isolated acanthosis nigricans, the face is not the most affected area. In the association of the two diseases, the peroral, paranasal, and eyelid areas are affected in almost all patients. It is possible that this happens because of the middle third facial hypoplasia that is characteristic of Crouzon patients.

The cause of acanthosis nigricans and its skin effects are not yet well established. In malignant acanthosis nigricans, there is secretion of a tumor polypeptide that is like epidermal growth factor. In association with endocrine disease and obesity, insulin resistance has been reported, such as that found in hyperandrogenism, Cushing syndrome, Addison disease, and hypothyroidism. Insulin resistance causes an excess of insulin, and when it binds with insulin and insulin-like receptors, it produces keratinocyte growth factor, leading to epidermal cellular proliferation. ${ }^{3}$

The association of Crouzon syndrome and acanthosis nigricans can be caused by a genetic defect that induces craniosynostosis and acanthosis nigricans. Recent biomolecular and genetic studies that analyzed the genotype of patients with both pathologies have revealed a mutation of the gene responsible for the fibroblastic receptor 3 transcription, located in chromosome $4 .{ }^{3,5}$ Studies of patients with isolated Crouzon syndrome revealed only a mutation of the gene responsible for the fibroblastic 2-receptor transcription, located in chromosome 10. These data support the idea that the association of Crouzon syndrome with acanthosis nigricans must be considered as a different pathology. ${ }^{4}$

In relation to the treatment of the craniofacial and dermatologic alterations found in these cases, craniosynostosis brought no improvement in the acanthosis, leading us to believe that those skin alterations are not secondary to the central nervous system changes that usually occur during the natural history of Crouzon syndrome. ${ }^{6}$

There are only 2 previously reported cases of the association of Crouzon syndrome with acanthosis nigricans in which the patients underwent skin resection on the eyelid. There is no report of complete blepharoplasty with canthopexy. Blepharoplasty and lateral canthal suspension, using the conventional cutaneous technique, is effective in producing aesthetic improvement of the cosmetic blemishes in the eylid area caused by this pathology, because this pro- 
cedure allows a greater amount resection of affected eyelid skin.

In the present case following blepharoplasty and lateral canthal suspension, the upper eyelid scars and the lateral portion of the lower ones became widened and depressed, possibly due the existing histological alterations. However, the obliquity of the eyelid fissure was improved, in spite of all the difficulties imposed by the case

\section{REFERENCES}

1. Nagase T, Nagase M, Hirose S, Ohmori K. Crouzon syndrome with acanthosis nigricans: case report and mutational analysis. Cleft Palate Craniofac J. 2000;37:78-82.

2. Koizumi H, Tomoyori T, Sato KC, Ohkawara A. An association of acanthosis nigricans and Crouzon syndrome. J Dermatol. 1992;19:1226.
3. Lapunzina P, Fernandez MC, Varela Junquera JM, Arberas C, Tello AM, Gracia Bouthelier R. Crouzon's syndrome with acanthosis nigricans. An Esp Pediatr. 2002;56:342-6.

4. Cohen MM Jr. Let's call it "Crouzonodermoskeletal syndrome" so we won't be prisoners of our own conventional terminology. Am J Med Genet. 1999;84:74. 
5. Meyers GA, Orlow SJ, Munro IR, Przylepa KA, Jabs EW. Fibroblast growth factor receptor 3 (FGFR3) transmembrane mutation in Crouzon syndrome with acanthosis nigricans. Nat Genet. 1995;11:462-4.
6. Breitbart AS, Eaton C, McCarthy JG. Crouzon's syndrome associated with acanthosis nigricans: ramifications for the craniofacial surgeon. Ann Plast Surg. 1989;22:310-5. 